\section{Viewing the corporate community as a knowledge network}

William Swan

Nigel Langford

Ian Watson and

Richard $\mathcal{F}$. Varey

\section{The knowledge context of the corporate community}

Knowledge and information can be appreciated within the context of Heath's (1994) "zones of meaning" that function within and between corporate communities. Heath (1994) characterizes zones of meaning as:

- interpretative schema or frames that people apply to their situation;

- generating meanings for the interpretation of actions relationships, and expectations;

- generating socially constructed realities; and

\section{The authors}

William Swan is in the School of Construction and Property Management, University of Salford, Salford, UK. Nigel Langford is in the BNFL Corporate Communication Research Unit, University of Salford, Salford, UK. Ian Watson is in the School of Construction and Property Management, University of Salford, Salford, UK. Richard J. Varey is in the BNFL Corporate Communication Research Unit, University of Salford, Salford, UK.

\section{Keywords}

Knowledge, Networks, Corporate communications

\section{Abstract}

The inter-organizational network is becoming an increasingly common form of organization. The majority of trade is carried out between organizations, rather than organizations and households. Many of these networks are concerned with the exchange of tangible goods. However, increasing numbers are concerned with the exchange of knowledge and all are dependent upon the role of knowledge in their activities. It is our assertion that with an understanding of the nature of knowledge, we may identify how, and why, certain network formations are adopted. It is asserted that links between organizations may be viewed as knowledge assets. The expression of multiple links within a corporate community may be regarded as a network of knowledge assets. From this conceptual framework, it may be possible to answer wider questions concerning the nature of networks established in the real world and how changes are wrought on a network over a period of time.

\section{Electronic access}

The current issue and full text archive of this journal is available at

http://www.emerald-library.com

Corporate Communications: An International Journal

Volume $5 \cdot$ Number $2 \cdot 2000 \cdot$ pp. 97-106

(C) MCB University Press $\cdot$ ISSN 1356-3289

\section{- enabling negotiated interaction in organizational and personal relationships.}

In this sense it is appropriate to view zones of meaning as expressions of knowledge assets facilitating:

the professional practice of developing and implementing communication rules and tools in order to enhance the dissemination, comprehension, acceptance and application of information in ways that will help to achieve an organization's goals (Gayeski, 1993).

The above conceptions are echoed by commentators such as Checkland and Holwell (1998), who refer to the process of creating organizational meaning as exclusively a human act:

It is the human being who can attribute meaning to the selected data which has been highlighted for attention, this being done in a context which may well be shared by many people but may also be unique to an individual (Checkland and Holwell, 1998).

Weick (1995) highlights the generation of organizational meaning and sensemaking as a

- grounded in identity construction;

- retrospective;

- enactive of sensible environments;

- social;

- ongoing;

- focused on and by extracted cues; and

- driven by plausibility rather than accuracy.

Finally, Choo (1998) views the development of organizational meaning as a necessary response to the reduction of: ambiguity in messages about the environment and to develop shared meaning among members in order for collective, purposeful action to take place (Choo, 1998). process that is: 
Viewing the corporate community as a knowledge network William Swan, Nigel Langford, lan Watson and Richard J. Varey

As organizations grow and segment through specialization, so do zones of meaning become differentiated and idiosyncratic. Heath (1994) sees that a key management task regarding differentiated zones of meaning is to strengthen the coupling between them to enable negotiation and compatibility in order to facilitate and ensure co-operation between the actors involved.

The following sections highlight the nature of that coupling, from the perspective of a network, focusing upon knowledge links between network actors, activities, and resources.

\section{The nature of knowledge}

Zones of meaning may be viewed as a form of knowledge asset. They are constructs or mental models shared between organizations. If this approach is to be adopted, it is important to have some understanding concerning the nature of knowledge.

Knowledge is currently being touted as the basis of future competitiveness, for example:

In the information age knowledge, rather than physical assets or resources is the key to competitiveness... What is new about attitudes to knowledge today is the recognition of the need to harness, manage and use it like any other asset. This raises issues not only of appropriate processes and systems, but also how to account for knowledge in the balance sheet (Moran, 1999).

Entrepreneurs are no longer seen as the owners of capital, but rather as individuals who express their tacit knowledge by "knowing how to do things" (Casson, 1997). Negroponte (1995) views the "atom" as having decreased in significance, and Boisot (1998) sees information as the key organizing principle of the organization, "information organizes matter". The introduction of information technology (IT) on a wide scale in the last 30 years has made the problem of capturing knowledge widely recognised, and brought to the forefront the issue of the management of knowledge assets (Davenport, 1997). The knowledge management function is spreading throughout organizations, in marketing, information management systems, and human resource management.

With knowledge now being viewed as a significant asset, the creation and sharing of knowledge has become an important factor within and between firms. Boisot (1998)
Corporate Communications: An International Journal

Volume $5 \cdot$ Number $2 \cdot 2000 \cdot 97-106$

refers to the "paradox of value" when considering the nature of knowledge, in particular its intangibility and inappropriability as an asset, and also the difficulty of protecting its value (Priest, 1994).

\section{Knowledge frameworks}

A common approach to considering knowledge often highlights its relationship to information in terms of difference. This perceived relational distinction between information and knowledge is not helpful and has led to the current confused preoccupation in the management literature with what is thought to be a clear distinction between "knowledge management" and "information management".

Although the relationship between information and knowledge may be seen as closely associated, it should be more appropriately seen in terms of a dynamic and interactive relationship. Over time, information facilitates the development of knowledge, which creates more information, which deepens knowledge, ad infinitum. For instance, Nonaka and Takeuchi (1995) state:

Information provides a new point of view for interpreting events or objects, which makes visible previously invisible meanings or sheds light on unexpected connections. Thus information is a necessary medium or material for eliciting and constructing knowledge.

Polyani (1967) and Choo (1998) have viewed this dynamic interactive relationship as part of the process of knowing which facilitates the capacity to act in context. The dynamic nature of this relationship is illustrated below in Figure 1.

Figure 1 The dynamic relationship between information and knowledge

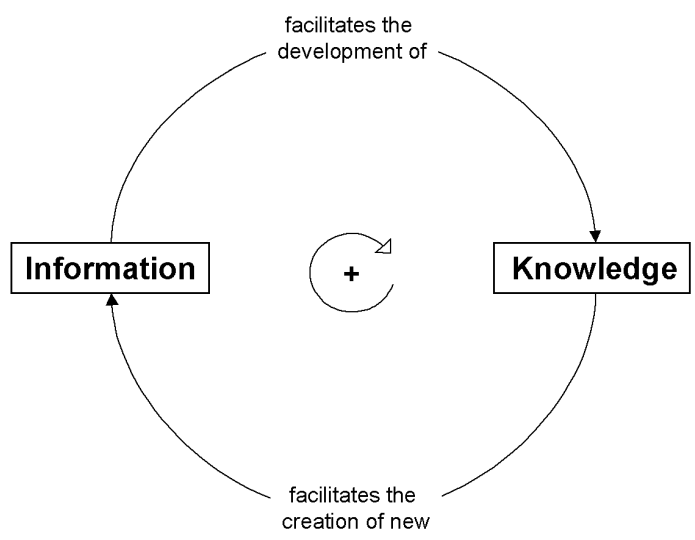


Viewing the corporate community as a knowledge network William Swan, Nigel Langford, lan Watson and Richard J. Varey

Similarly, to look at information purely in terms of the degree to which it has been processed, i.e. the data, information, knowledge hierarchy (Davenport, 1997; Checkland and Holwell, 1998) oversimplifies the complex relationship between the three intangibles. Stewart (1997) notes:

The idea that knowledge can be slotted into a data-wisdom hierarchy is bogus, for the simple reason that one man's knowledge is another man's data.

Boisot (1998) relates these states of intangibles in a manner that supports and adapts to phenomenological and constructivist approaches of Piaget. The categories defined by Boisot, and their interactions, may be seen in Figure 2. It is important to note the feedback element, which illustrates the dynamic and interactive relationship of information and knowledge as a positive feedback loop.

Data is discrimination between physical states - black, white, heavy, light - that may or may not convey information to an agent. Whether it does or not depends on the agent's prior stock of knowledge. The states of nature indicated by red, amber and green traffic lights may not be as informative to a Kung bushman of the Kalihari, for example, as are certain patterns in the soil that would indicate the presence of lions nearby. Thus, whereas data can be characterised as a property of things, knowledge is a property of agents predisposing them to act in particular circumstances. Information is that subset of the data residing in things that activates an agent - it is filtered from the data by the
Corporate Communications: An International Journal Volume $5 \cdot$ Number $2 \cdot 2000 \cdot 97-106$

agent's perceptual or conceptual apparatus (Boisot, 1998).

Expanding upon the work of Nonaka and Takeuchi (1995), Boisot (1998) has formulated a conceptual three-dimensional space called the "I-space", the "information space". The I-space is delineated by three axes, as illustrated in Figure 3. Each axis represents the distribution of the properties of information arrayed in order of increasing or decreasing magnitude. The properties that are instrumental in preventing or facilitating diffusion are the degree to which data has been "abstracted" and "codified", characterised by Boisot as follows:

\section{Codification}

- Can be thought of as a process of giving form to phenomena or to experience.

Figure 3 Informational activities within the I-space

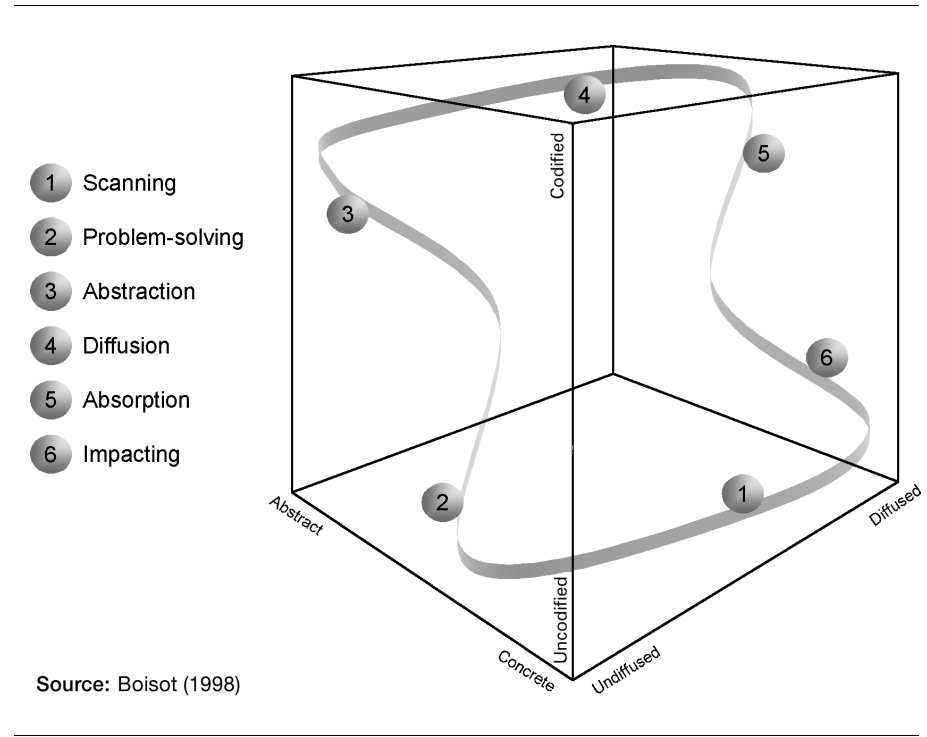

Figure 2 Data, information and knowledge

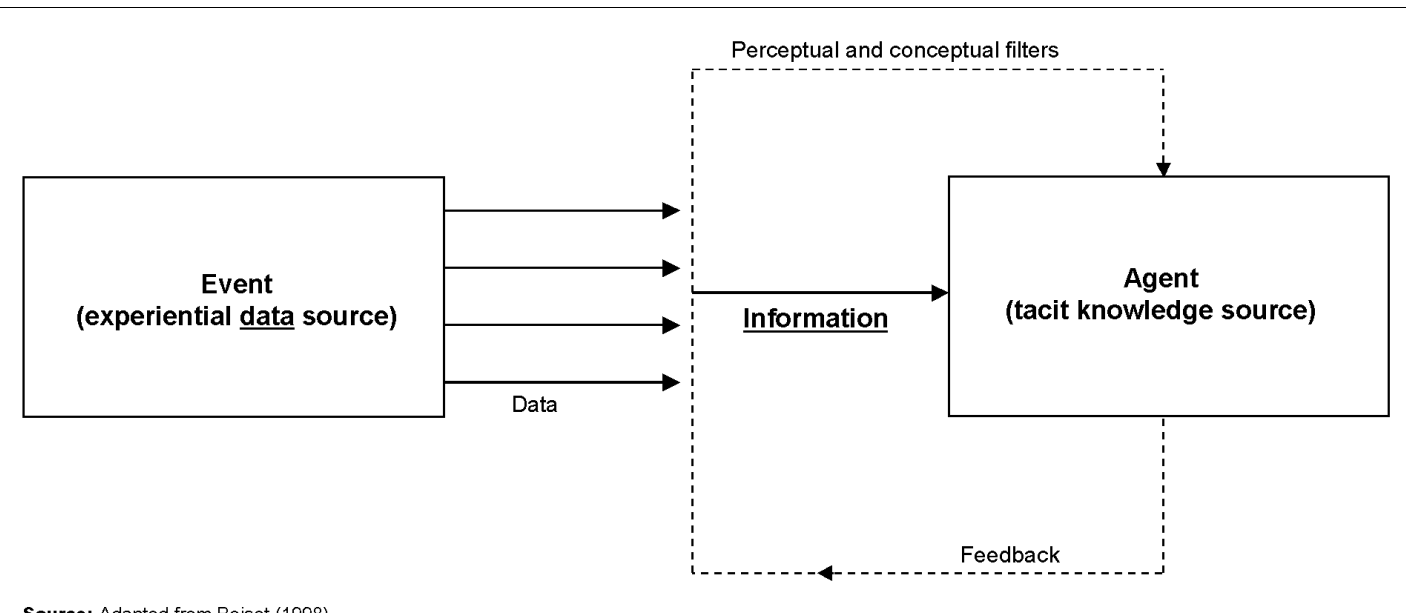


Viewing the corporate community as a knowledge network William Swan, Nigel Langford, lan Watson and Richard J. Varey

- Creates perceptual and conceptual categories that facilitate the classification of phenomena.

- Effective codification is partly a matter of intellectual and observational skill. An ability to discern contour and form in the data of experience.

- Depends upon the complexity of phenomena that require partitioning into categories. The larger the number of distinctive attributes associated with a phenomena the more problematic the act of codification.

- Generates both cognitive and behavioural commitment, i.e. percepts.

- Is a procedure for shedding surplus data.

\section{Abstraction}

- Is the process of discerning the structures that underlie the forms evoked by codification.

- If codification allows us to save on dataprocessing resources by allowing us to group the data of experience into categories, abstraction allows us to realize further savings in data processing by minimizing the number of categories that we need to draw on for a given task.

- Works by teasing out the underlying structure of phenomena relevant to our purpose.

- Requires an appreciation of cause-andeffect relationships to an extent that simple acts of codification do not.

- Allows one to focus on the structures, causal or descriptive, that underlie data.

- Generates concepts rather than percepts. Where percepts generate their economies by maintaining clarity and distinction between categories, concepts do so by revealing which categories are likely to be relevant to the data processing task.

- Is a form of reductionism; it works by letting the few stand for the many.

Abstraction of information is determined by its relationship with the real world. The "concrete" is a knowledge asset that is grounded in the real world, such as an individual's capability to carry out a certain activity. An abstraction of the concrete would be the establishment of general rules or procedures for all activities associated with the activity in question.

Abstraction then, works by teasing out the underlying structure of phenomena relevant to our purpose. It requires an appreciation of cause
Corporate Communications: An International Journal

Volume $5 \cdot$ Number $2 \cdot 2000 \cdot 97-106$

and effect relationships to an extent that simple acts of codification do not ... It [an abstraction] generates concepts rather than percepts. Like percepts, concepts are devices that economise on data processing. Yet whereas percepts achieve their economies by maintaining a certain clarity and distinction between categories, concepts do so by revealing which categories are likely to be relevant to the data processing task (Boisot, 1998).

Abstraction like codification is an organizing principal. However, there is a distinction between the two; codification "organizes knowledge", abstraction "draws off" the important elements of certain knowledge.

Standards and methodologies are themselves forms of codification and abstraction that represent significant investment, and it may prove difficult and costly to replace them with technologically or methodologically superior systems. The choices made regarding modes of method or standard can be viewed as sources of competitive advantage (Boisot, 1998).

Abstraction and codification work in tandem to reduce the data processing load imposed upon a data processing agent, i.e. they act as economizing processes facilitating the flow of information, and in doing so facilitate the extension of the diffusion of information within a data processing population.

Such a population need not consist of individual human beings. It could be made up of firms, industries or even countries. All that we require is that each member of a population exhibit a similar general capacity for receiving, processing and transmitting data (Boisot, 1998).

The notion of a firm's "population" or publics reflects the extent of the firm's networked environment, i.e. those individuals and organizations with which the firm conducts business activity.

There is some difficulty in determining the extent of the networked boundary, and Axelsson and Easton (1992) conclude that it is, essentially, arbitrarily determined by the nature of the study of the knowledge flows.

Codification and abstraction facilitate the diffusion of information. The degree of diffusion

... establishes the availability of data and information for those who want to use it. It does not measure adoption: information may be widely diffused and yet remain unused" (Boisot, 1998). 
Diffusion of information represents the availability of information within a defined population. The factors that support diffusion are an expansion of the semiotic levels discussed by Shannon and Weaver (1949), Roszak (1986) and Liebenau and Backhouse (1990). These include:

- Empiric - physical (technical).

- Syntactic - linguistic.

- Semantic - content.

- Pragmatic - contextual.

The ability of information to flow across these channels within a network setting is an aid to its diffusability. For example, it is considered that the failure of many IS projects is caused by the inability to appreciate the nature of the communications structure, particularly at the pragmatic level (Davenport, 1997; Davenport and Prusak, 1997; Landauer, 1995).

It is established that the creation of knowledge is a dynamic process. The cycle in Figure 3 shows the passage of a specific type of knowledge throughout a given population. If, for example, we were to consider a population of scientists, the point in the lower corner of the I-space (diffused, concrete, uncodified) may indicate some intuitive thoughts and observations held by a specific individual concerning a subject. An experiment may be designed and results published. There may be a generation of supporting and opposing papers and hypotheses - knowledge may eventually progress to a simple equation located at the top corner of the I-space (diffused, abstract, codified), clearly available and easily diffused, without friction. The reality of such knowledge processes is that many of them are subject to considerable frictions that either limit or completely curb knowledge processes within an organization. This form of knowledge asset cannot be directly related to the knowledge networks we are regarding here. Zones of meaning and contexts are not as easily transferable, or predictable, as other forms of knowledge asset.

\section{Cultural considerations}

The presence of cultural proclivities within an organization have been well documented, but cultural proclivity within a knowledge network, i.e. between different zones of meaning, account for significant differences in network structure. Boisot (1998) views the relationship of knowledge and culture as problematic, in so far as:

only a small part of what we call cultural knowledge gets embedded in technologies and artefacts. A large part is embodied in social processes, institutional practices, and traditions, many of which are carried around in people's heads.

This being the case, the cultural confluence of organizations within a knowledge network requires significant effort to overcome, or combine, social and institutional practices in order to develop trustful relationship between participants. Boisot (1998) has identified four institutional "archetypes" that embody separate cultural knowledge and the knowledge utilizing characteristics outlined above. Boisot's institutional types are as follows:

\section{Bureaucracies}

- No necessity to share cultural knowledge.

- Information is codified and abstract.

- Information diffusion is limited and under central control.

- Relationships are impersonal and hierarchical.

- Co-ordination is hierarchical.

\section{Markets}

- No necessity to share cultural knowledge.

- Information is codified and abstract.

- Information is widely diffused with no controls.

- Relationships are impersonal and competitive.

- Co-ordination is horizontal through selfregulation.

\section{Clans}

- Necessity of sharing cultural knowledge.

- Information is uncodified and concrete.

- Information diffusion is limited to faceto-face relationships.

- Relationships are personal and nonhierarchical.

- Co-ordination is horizontal through negotiation.

Fiefs

- Necessity of sharing cultural knowledge.

- Information is uncodified and concrete.

- Information diffusion is limited to faceto-face relationships. 
Viewing the corporate community as a knowledge network

William Swan, Nigel Langford, lan Watson and Richard J. Varey

- Relationships are personal and hierarchical (feudal/charismatic).

- Co-ordination is hierarchical.

Given the above institutional types, it can be seen that the characteristic of "sharing cultural knowledge" embodied within clans and fiefs facilitates the exchange of different types of knowledge and information between zones of meaning, allowing a firm to "develop an organizational capacity that reaches beyond its corporate boundary" (Boisot, 1998). Where knowledge of some types may be embodied in prices (as in markets), or in rules (as in bureaucracies), it is clear that complex contextual transactions will require the clan or fief model to successfully exchange complex forms of knowledge.

\section{Networks - defining the constituent elements of a network}

A network is typically defined in terms of:

Actors - who occur at several levels within the organization and are characterized by:

- Performing and controlling activities.

- Developing relationships with other actors.

- Base their degree of control of resources depending upon direct or indirect ownership and relation within the network hierarchy.

- Are goal oriented. The general goal of actors is to facilitate control within their network boundary. There are different levels of knowledge within the population.

Activities - where there are two key types of activity cycles (tightly or loosely coupled) and characterized by:

- Transformational activities where resources get "changed".

- Transfer activities move control of resources from one actor to another. These types of activities link actors together.

Resources - are utilized within activity processes and are cyclical in nature:

- Transformation and transfer activities require resource utilization.

- Resources are combined and the act of combination requires resources.

Actors are connected together via network links. Links are formed using the semiotic
Corporate Communications: An International Journal Volume $5 \cdot$ Number $2 \cdot 2000 \cdot 97-106$

model discussed earlier, i.e. empirics, syntactics, semantics and pragmatics. Empirics refers to the technical level of communication, the ability to physically make the communication via whatever medium. Referring to Figure 4, this could reflect the ability to perceive data through physical media; this could encompass face-to-face communication or complex computer systems. Syntactics refers to the linguistic ability to communicate, i.e. whether or not there is a shared structure to the language. Semantics is concerned with the content of the message. This is shown in Figure 4 by the flow of information. Pragmatics is the context of the communication, and existing zones of meaning. The participants in a communication act will interpret a data source in the context of their own existing knowledge (Liebenau and Backhouse, 1990; Roszak, 1986). The links are layered from empiric to pragmatic. The lower levels must be satisfied for a communication to be successful - communication must be physically possible, at the empiric level, before issues of context, pragmatics, can come into play.

Actors create and maintain relationships with each other and require knowledge of other actors within the network in order to do so. Actors are the key constituents in strengthening the coupling between differentiated zones of meaning and enable negotiation, co-ordination and compatibility in order to facilitate collective and purposeful action to take place (Choo, 1998). Activities

Figure 4 A typical network construct

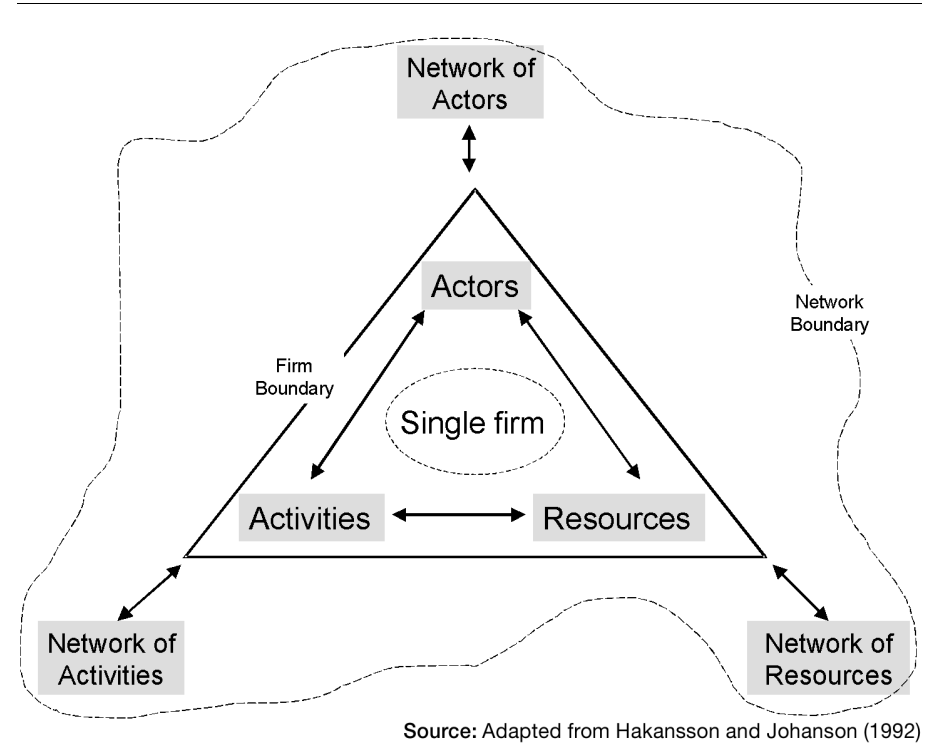


are those processes in which actors coordinate the utilization of resources. The cyclical relationship between actors, activities and resources is illustrated in Figure 5.

The cohesive forces that enable the coupling of zones of meaning within a corporate community take several forms, all of which depend upon the development of joint knowledge constructs at the interface of each zone. Typically these will include a functional interdependence between zones, and a power/authority arrangement between zones whereby resources are controlled, or a transactional contract.

Another conception of knowledge networking within and between stakeholders in the corporate community is Wikström and Norman's (1994) conception of the "knowledge based value star" (Figure 6).

Figure 5 Network relationships

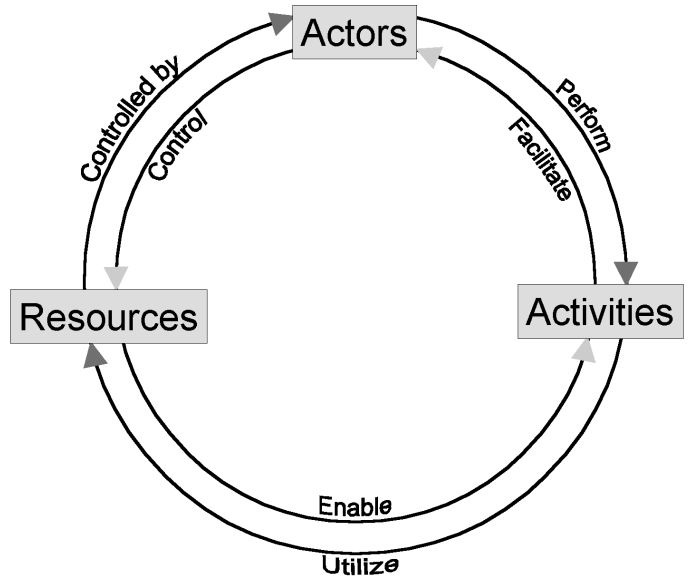

Value-creating knowledge processes occur at the interfaces or the points of convergence of knowledge flow. Sources for this flow are facilitated through the creation of information-sharing networks focused on three modes of knowledge development. The modes, according to Wikström and Norman (1994), are:

- Generative - knowledge developed and created through joint activities involved with solving problems.

- Productive - knowledge developed and accumulated through the creation of products and services. This knowledge is re-productive in the sense that it may be applied repeatedly but within a different guise.

- Representative - knowledge made available to suppliers for example, concerning their own value creating processes.

Knowledge-based value stars are reciprocally dynamic in character. Being a "member" of a knowledge network such as a value star actively discourages the hoarding of information. Being non-reciprocal is easily identifiable given that it goes against the whole nature of the network and results in a network-wide depletion of trust and eventual exclusion from network activities.

Alternatively, the growth of the knowledge network enables organizations to develop from being a network conduit to becoming the focus of knowledge flow. Normann and Ramirez (1993) refer to this focus as a

Figure 6 The network as a knowledge creating value star

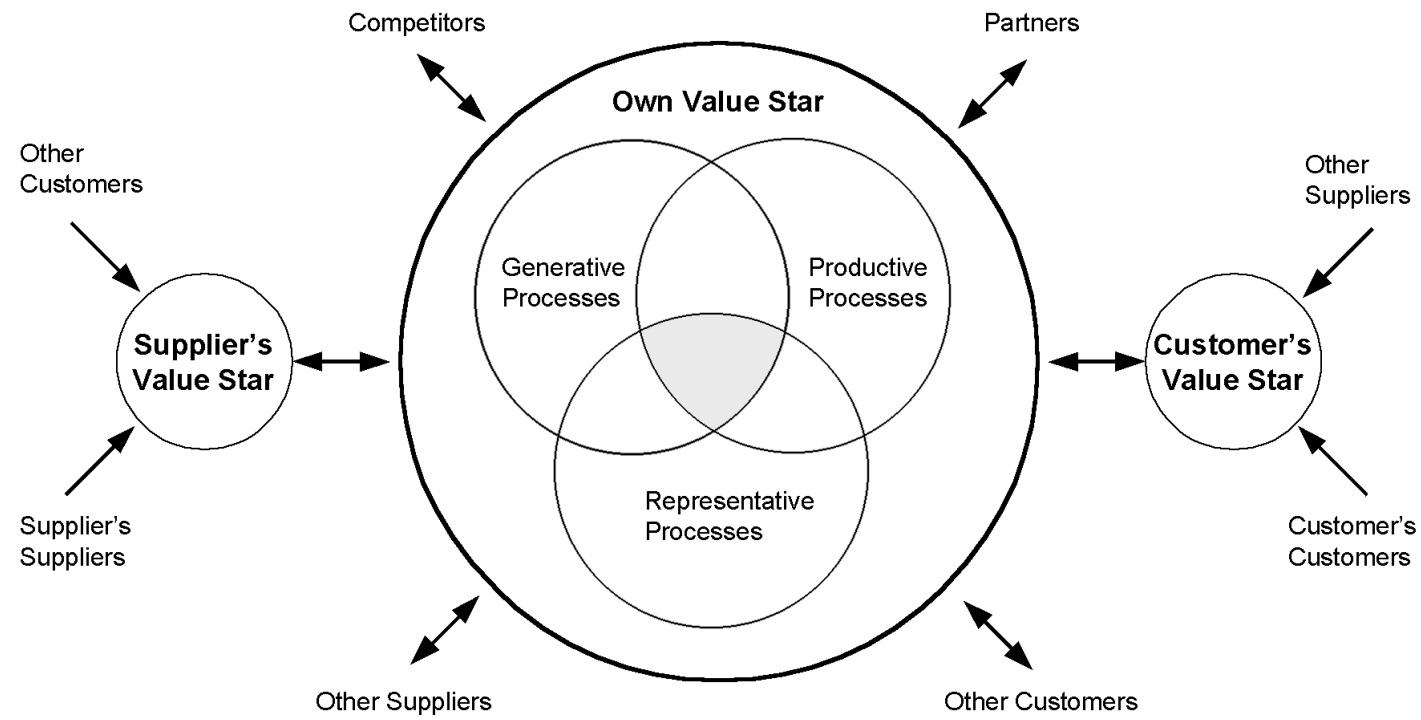

Source: Wikstrom and Norman, 1994 
Viewing the corporate community as a knowledge network William Swan, Nigel Langford, Ian Watson and Richard J. Varey

reflection of "knowledge density" which they define as:

a measure of the amount of information, knowledge, and other resources that an economic actor has at hand at any moment in time to leverage his or her own value creation.

They view the significance of knowledge density as residing in the development of trust and the subsequent access this relationship provides to the customer's and/or supplier's value creating activities.

Badaracco (1991) refers to the development of these types of relationships as "alliances" that are brought about through the implementation of "knowledge links" focusing upon the special character of embedded knowledge that resides primarily:

in the specialized relationships among individuals and groups and in the particular norms, attitudes, information flows and ways of making decisions that shape their dealings with each other.

Badaracco (1991) makes a clear distinction between "product links" and "knowledge links". Where product links may be thought of as similar to the activities of supply chain management, knowledge links are primarily concerned with enhancing and extending current capabilities, i.e.:

- Learning and creating knowledge.

- The development of intimate working relationships spanning organizational boundaries.

- Working with partners within and outside the industry.

- Developing joint long-term strategic capabilities.

The above characteristics once more emphasise the importance of relationships and the development of trust within the corporate network. Badaracco (1991) talks of designing work and social structures to "facilitate technical and personal empathy". Similarly, Choo (1998) views the utilisation and development of networked knowledge structures as much as a social as a technical process where the pace and scope of knowledge mobilisation depends upon an organizational culture that promotes overlapping behaviours such as information sharing and joint experimentation. Larson (1992), too, emphasises the need for trust, a sense of mutual obligation and the free flow of information within any network. Social control is a crucial element where selfregulation occurs within a moral dimension
Corporate Communications: An International Journal Volume $5 \cdot$ Number $2 \cdot 2000 \cdot 97-106$

and feedback processes are jointly determined by and diffused across the multiple participants within the network.

The network of the corporate community is essentially the information population with which we are concerned at this time. A network is essentially composed of nodes, links, a boundary, and an environment, taking a systemic approach to the view of the network (Ebers, 1997).

In considering the network as a system, it is important to consider the system's relationship to its environment and, implicit in this, the boundary of the system. In this sense, knowledge networks can be viewed as an open systems, as defined by Bertalanffy (see Malhotra, 1996, for explanation).

The boundaries of such a system are problematic and will vary depending upon the purposes for which the boundary is being draw (Axelsson and Easton, 1992).

The boundary of network is essentially arbitrary, and is generally determined by those factors over which the network has control. If a factor remains wholly outside the control of the network then it is considered an environmental factor. There are some factors that could be considered to lie across the dividing line, with the network actors having only partial control.

\section{An example of a knowledge network}

In a current study of a knowledge network, an inter-organizational network dedicated to the creation and dissemination of knowledge assets, this knowledge network approach has revealed certain aspects of the creation of zones of meaning and their importance in the functioning of knowledge networks of this type. The Construct-IT network (administered at the University of Salford) was established in 1995 to improve the level of IT use within construction companies, in line with the recommendations of the Latham Report (1994). The network membership is made up of contractors, clients, software and hardware suppliers, universities and research bodies, and professional organizations, such as lawyers and accountants. Separately, with these organizations it is clear that there are very different cultures at work here.

The network structure of the Construct-IT organization could be viewed as a mixture between the fief and clan structures. The types of knowledge exchanged are semantically 
Viewing the corporate community as a knowledge network William Swan, Nigel Langford, lan Watson and Richard J. Varey

unstructured and relatively complex. At the initial stages, some participants indicated that they were unsure of the "purpose" of the organization, and some confusion concerning the activities of the network in the early stages resulted. Through exchange interactions, a zone of meaning was created. There was clear understanding among participants of what the purpose of the network now was, and hence the knowledge activities being undertaken were now considered a better reflection of what the participants believed they required. This is not because the network changed the entirety of what it was doing, although some changes were undertaken, but largely because the participants, through discourse between themselves and the controllers of the network, had created some shared meaning.

Many members joined on the basis of little or no knowledge of the subject area. As they have absorbed knowledge concerning the domain area they are more able to make decisions concerning the control of the resources within the network. The domain was not framed in the early stages of the network operation. It remained in the concrete arena of the individual perception of individual projects carried out within organizations. The use of a "framing device" was adopted at the early stages. A framing device could be viewed as a form of codification to organise the subject area. The issues were abstracted from specific problems of individual projects and framed within a generic understanding of construction processes across the board (Kagioglou et al., 1998). This shared codification allowed for the generation of general knowledge between the participants. This shared understanding of the problem domain also represents a shared zone of meaning. For the IT participants within the project, their understanding of the situation may have been considered as abstracted from the problems of construction. The method of codification allowed a frame of reference for discourse, allowing them to view the problem in a more concrete form.

Interviews with participants have revealed that zones of meaning (Heath, 1994) were often not consciously developed as such, but rather were a product of repeated exchanges within a network. The network formation has grown as a response to individual willingness to participate. A common comment was that participants "got out what they put in". Repeated exchange created a core of organizations at the centre of the network,
Corporate Communications: An International Journal Volume $5 \cdot$ Number $2 \cdot 2000 \cdot 97-106$

with other organizations willing to follow the decisions made by these central members. Some of these members expressed willingness to participate more fully as they found a "clearer idea" of the domain. Although the members did not consciously develop these zones of meaning, the ability to "talk the same language" was recognised as an important factor. There was an understanding that the creation of these zones of meaning had led to the current perceived success of the network, and had enabled continued exchange.

\section{Conclusion}

A network, according to Ebers (1997), is determined by exchange relationships between nodes. This may encompass markets, joint ventures, or "club" networks. In the authors' model it encompasses the zones of meaning present within the corporate community.

While networking can take different forms, all forms are characterised by recurring exchange relationships among a limited number of organizations that retain residual control of their individual resources yet periodically jointly decide over their use (Ebers, 1997).

Knowledge networks are dynamic. Their structure and boundaries will change over time as actors are included or excluded from the network, and activities and resources are superseded or become obsolete. For example, a knowledge network that begins life as a loosely affiliated trading group or organizational public, may eventually turn into market archetype, heavily codified and structured by information technologies. This can be seen in the Construct-IT network where the initial "sense-making" approach concerning the creation of a shared context has given way to task-based interest groups. While the goals of the interest groups are often with a view to specific knowledge deliverables, these activities reinforce the shared zone of meaning, and thus benefit network operations.

Where the knowledge is constantly being reevaluated and thereby will remain semantically complex and difficult to codify, the networks will remain in the fief and clan formation, where redundancy of communication allows more complex communication to be "negotiated" through discourse rather than "transmitted" in a highly codified form. There is theoretically the possibility of hybridised forms of networks. 
A corporate community within the context of Heath's (1994) zones of meaning can be viewed as a knowledge network. In the case of networks for creation and sharing of knowledge the more detailed, structured approach certainly allows for greater potential management of the knowledge processes within a coherent framework. Both viewpoints are valid, as the concepts of shared meaning (contexts) and framing devices (modes of codification) have a critical impact on the knowledge processes within such a network. The process-based approach supported by Boisot (1998) indicates that a zone of meaning is dynamic. At a specific point in time it may be viewed as an asset to the network, but an unwillingness to accept the dynamic nature of these zones and their constant re-evaluation by participants and external observers in relation to new data, can lead to calcification of a mind-set and the asset can become a source of rigidity (Leonard-Barton, 1995). This approach to the destruction of network assets mirrors Schumpeter's (1934) notion of creative destruction and allows for the development of, and engagement with, a full knowledge cycle (Boisot, 1998). The term "community", as used here, carries specific intended meaning (Etzioni, 1994).

\section{References}

Axelsson, B. and Easton, G. (1992), Industrial Networks, a New View of Reality, Routledge \& Kegan Paul, London.

Badaracco, J.L. (1991), The Knowledge Link - How Firms Compete through Strategic Alliances, Harvard Business School Press, Boston, MA.

Boisot, M. (1998), Knowledge Assets - Securing Competitive Advantage in the Information Economy, Oxford University Press, Oxford.

Casson, M. (1997), Information and Organization. A New Perspective On the Theory of the Firm, Clarendon Press, Oxford.

Checkland, P. and Holwell, S. (1998), Information, Systems and Information Systems: Making Sense of the Field, John Wiley \& Sons, London.

Choo, C.W. (1998), The Knowing Organization, Oxford University Press, Oxford.

Davenport, T.D. (1997), Information Ecology: Mastering the Information and Knowledge Environment, Oxford University Press, Oxford

Davenport, T.D. and Prusak, L. (1997), Working Knowledge, Harvard Business School Press, Boston, MA.

Ebers, M. (1997), The Formation of Inter-organizational Networks, Oxford University Press, Oxford.
Etzioni, A. (1994), The Spirit of Community: Rights, Responsibilities and the Communitarian Agenda, Simon \& Schuster, New York, NY.

Gayeski, D. (1993), Corporate Communications Management: The Renaissance Communicator in Information-age Organizations, Focal Press/ Heinneman, Boston, MA.

Hakansson, H. and Johansson, J. (1992), "A model of industrial networks", cited in Axelsson, B. and Easton, G. (Eds), Industrial Networks: A New View of Reality, Routledge \& Kegan Paul, London.

Heath, R.L. (1994), Management of Corporate Communications, Lawrence Erlbaum, Hillsdale, NJ.

Kagioglou, M. et al. (1998), A Generic Guide to the Design and Construction Process Protocol, University of Salford.

Landauer, T.K. (1995), The Trouble with Computers, MIT Press, Cambridge, MA.

Larson, A. (1992), "Network dyads in entrepreneurial settings: a study of the governance of exchange relationships", Administrative Science Quarterly, Vol. 37 No. 1, pp. 76-105.

Latham Report (1994), Constructing the Team, HMSO London.

Leonard-Barton, D. (1995), Wellsprings of Knowledge Harvard University Press, Boston, MA.

Liebenau, J. and Backhouse, J. (1990), Understanding Information: An Introduction, Macmillan, London.

Malhotra, Y. (1996), "Organizational learning and learning organizations", www.brint.com/papers/orglrng.htm

Moran, N. (1999), "Becoming a knowledge-based organization", Financial Times Survey - Knowledge Management, 28 April.

Negroponte, N. (1995), Being Digital, Hodder \& Stoughton, London.

Nonaka, I. and Takeuchi, H. (1995), The KnowledgeCreating Company: How Japanese Companies Create the Dynamics of Innovation, Oxford University Press, Oxford.

Normann, R. and Ramirez, R. (1993), "From value chain to value constellation: designing interactive strategy", Harvard Business Review, Vol. 71 No. 4, pp. 65-77.

Polyani, M. (1967), The Tacit Dimension, Routledge \& Kegan Paul, London.

Priest, W.C. (1994), "An information framework for the planning and design of 'information highways"', Berkley information site.

Roszak, T. (1986), The Cult of Information, Pantheon Books, New York, NY.

Schumpeter, J.A. (1934), The Theory of Economic Development, Oxford University Press, Oxford.

Shannon, C.E. and Weaver, W. (1949), The Mathematical Theory of Communication, University of Illinois Press, Urbana, IL.

Stewart, T.A. (1997), Intellectual Capital, Nicholas Brealey, London.

Weick, K.E. (1995), Sensemaking in Organizations, Sage Publications, Thousand Oaks, CA.

Wikström, S.R. and Norman, R. (1994), Knowledge and Value: A New Perspective on Corporate Transformation, Routledge \& Kegan Paul, London.

\section{Further reading}

Zuboff, S. (1988), In the Age of the Smart Machine: The Future of Work and Power, Basic Books, New York, NY. 